\title{
Design of Estimator for Computing Yaw and Pitch for a Twin Rotor MIMO system
}

\author{
Santhosh K V, Preeti Mohanty \\ Centre for Cyber Physical System, Department of Instrumentation and Control Engineering, Manipal \\ Institute of Technology, Manipal Academy of Higher Education, Manipal, \\ India
}

Received: April 10, 2020. Revised: May 30, 2020. 2nd-Revised: June 17, 2020.

Accepted: June 23, 2020. Published: June 24, 2020.

\begin{abstract}
Performance of any system is identified through the observation of significant system parameters. Required parameters have to be measured using suitable sensors. But in some scenarios, it is difficult to measure some of the parameters due to issues in the placement of sensors. In such cases, estimators are developed to measure the parameters indirectly. In this paper, an attempt is made to develop an estimator to monitor the value of pitch and yaw of a twin-rotor multi input multi output system. The observer is developed using two methods one using Luenberger's equations and the other using an Artificial Neural Network (ANN). For training the neural network model, the backpropagation algorithm is used. Tests have been conducted to analyze and compare the behavior of both observers. From the results, it is evident that a Luenberger observer performs better when sufficient system information is available and ANN observer performs better when inadequate system information is available.
\end{abstract}

Keywords-Artificial Neural Network, Estimator, Observer, soft sensor, TMRS.

\section{INTRODUCTION}

I NVENTION of helicopters were considered as the evolutionary step towards vertical lift vehicles. Study of helicopter system is vital in many ways to understand the behavior of aircrafts. Since, design and implementation of the helicopter will not be viable solutions for many institutes, a TRMS as shown in Figure 1 is implemented to mimic the operation of the same. Several researchers have reported works on understanding this vital component of an aircraft system.

A lot of work have been reported for measurement and control of a TRMS system like in [1], a technique is reported for control of pitch channel in a TRMS using nonlinear inversion control model programmed using $\mathrm{ANN}$ and genetic algorithms. Design of a neural Proportional+ Integral+ Differentiator (PID) controller for control of pitch and yaw in TRMS was developed in [2]. PID controller was tuned using real value genetic algorithm. Paper [3] discusses the design of controller for stabilizing the TRMS using a radial basis function neural network algorithm. In [4], a setup is implemented for virtual and remote control practices in a three Degree of Freedom (DOF) quadrotor. TwinCAT Programmable Logic Controller (PLC) are used as the controllers and applet as remote laboratory front-end. Paper [5] discusses a robust tracking system developed using an integral sliding mode controller for a TRMS. Simultaneous estimation of system fault and sensor fault is discussed in [6]. The method discussed reduces the exogenous disturbances to a predefined level apart from the ability to perform simultaneous estimation. Paper [7] explored the concept of shifting linear quadratic control which considered the existence of constraints in the system. Two constraints namely, algebraic constraints between variables of the system and constraints depending on the input and state variables were considered. In [8], a hybrid controller containing a second order data driven Model Free Control (MFC) and Takagi-Sugeno Fuzzy (TSF) logic controller for nonlinear Multi Input-Multi Output twin rotor aerodynamic systems was reported.

A comparison of the performance of the Control System Structure (CSS) using the second order MFC-TSF controller and CSS using the MFC controller was performed. Paper [9] discusses the Integral Quadratic Constraints (IQC) approach, for effective systematic analysis of robust stability. To make IQC analysis tools easily accessible, an overview of three measures namely (i) general setup and basic IQC theorem, (ii) a survey of multipliers based on the linear matrix inequality constraints and (iii) an explanation on application of the tools is done. An analytical method for based on Bode's ideal transfer function for tuning the parameters of fractional order PI controllers (FOPI) is discussed in [10]. In [10], to attain the closed loop transfer function the factors of the FOPI controller 
were tuned. It was found that for low and medium frequency ranges, the robustness of the system is better in FOPI controller with compared to the PI controller with similar configuration.

Paper [11] reports a design and experimental of robust controller for multi input multi output plants. External disturbances and parametric uncertainties were the factors considered for measuring the quantized output. A control strategy for TRMS is discussed in [12], to reach preferred positions in two degrees of freedom, a control strategy built on the coupling of a fuzzy logic control using sliding mode controller. In [13], identification, modeling and control of TRMS for a quasi linear parameter is reported. Nonlinear model is converted in to a quasi LPV system. Paper [14] reported a different nonlinear internal model control approach. The performance and robustness can be improved by varying the control based on flatness property. This enables the model to work in a closed loop structure. Two nonlinear model predictive control algorithms are projected in [15] based on neural networks intensified reactors. One control algorithm is by means of nonlinear-optimization and the other using local linearization.

Paper [16], reports a multi variable nonlinear control oriented model for a twin rotor aerodynamic system. Using Lagrange's equations, a mathematical description for a multibody system is derived. A multi-variable integral sliding mode control using the resulting state-space representation is designed which tracks the preferred trajectories for azimuth angle and pitch angle. Paper [17] describes data-driven techniques which are applied on a single MIMO controller and two separately designed single-input-single-output controllers. The data driven techniques projected are model-free adaptive control, model-free control and virtual reference feedback tuning techniques. A model predictive control strategy designed for drinking water networks is discussed in [18] which considers the system and component reliability. In [19], an observer centered control method for a two input two output plant is reported. The plant is affected by lumped disturbance which consists of undesired effect of cross couplings, parametric uncertainties and external disturbances.

Paper [20] discusses the development of a model predictive control approach built on a neural network Wiener model. The paper also discusses its application on an intensified continuous reactor. The Wiener model can be grouped into two parts: a linear state space identified model and a local linearization of a neural network model. A laboratory model of a Twin Rotor MIMO system is described in [21] which was created by Feedback Instruments Ltd. The system which looks like a helicopter is made up of two rotors. In [22], a model of the Mamdani type fuzzy two input, two output proportional integral or proportional differentiator controller is reported. The fuzzy controller consists of two fuzzy sets for each input variables, five fuzzy set for each output variable, five linear control rules, AND operator, OR operator and height defuzzification strategy. Paper [23] talks about two model free sliding mode control structures. On comparison with a model free intelligent proportional integral control system structure in regard to performance improved performance is found. Paper [24] describes the design of passivity-based controllers which makes use of memristor. Making use of the passive property of memristor incorporated in the target dynamics. In [25], a linear parameter varying model is reported meant for fixed-wing unmanned aerial vehicles. The objects obtained includes its agility and high performance.

From the literature survey it is clear that a lot of study is reported on TRMS systems, with the focus mainly on the control aspect. A few literature also reports design of sensing technique for measurement of variables like pitch, yaw and roll. Few literature have also focused on the fault tolerant control system, the proposed work makes an attempt to design estimator to measure parameters like pitch yaw in TRMS under the condition of faulty sensors.

The work is organized such that the background study is carried out in first section followed by description of the practical system in Section-II. Section-III reports the methodology of the proposed work. Section-IV discusses the results obtained by the ANN based and observer based techniques. Finally, Section-V discusses the conclusion of the work.

\section{SYSTEM DESCRIPTION}

Twin rotor multi input and multi output system from feedback instrument Pvt ltd is used for carrying the experiment in the proposed work. Schematic of the proposed system is shown in Figure 1 [26], the TRMS model similar to the helicopter model. The experimental model consists of beam pivoting on its base at both of its end. The beam is connected with two propeller which are driven by two DC motors. The beam is fabricated in a way such that the beam can traverse in a circular pattern along the axis. A counter weight is provided at the center, to keep the system in the state of equilibrium. The system is designed in a way such that when motors are switched off the end with main rotor in lowered.

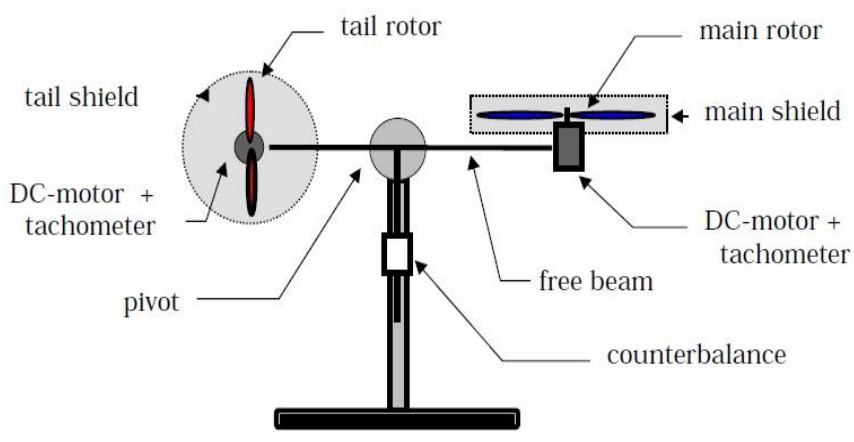

Fig. 1 Schematic of the proposed system

Supply voltage of $230 \mathrm{~V} \mathrm{AC}$ is used to drive the motor to so 
as to control the TRMS parameters. Position angles and angular velocity of the rotor are the two signals which are measured. Software codes are used to reconstruct the angular velocities from the position angles of the beam by differentiation and filtering. Fixed angle of attack of a rotor and aerodynamics are controlled by varying speed of the motor. Cross coupling is observed in the movement of the rotors, with each rotor affecting both angle positions. The two propellers are driven by DC motors and control of the system are the supply voltages for the motor. Measured signals are positioned in the beam in the space that is two position angles which are measured by rotary optical encoders, mounted on each of the rotor shaft. The optical encoder to measure the rotation of the rotor is of incremental type. Figure 2 shows the laboratory model used in the proposed work.

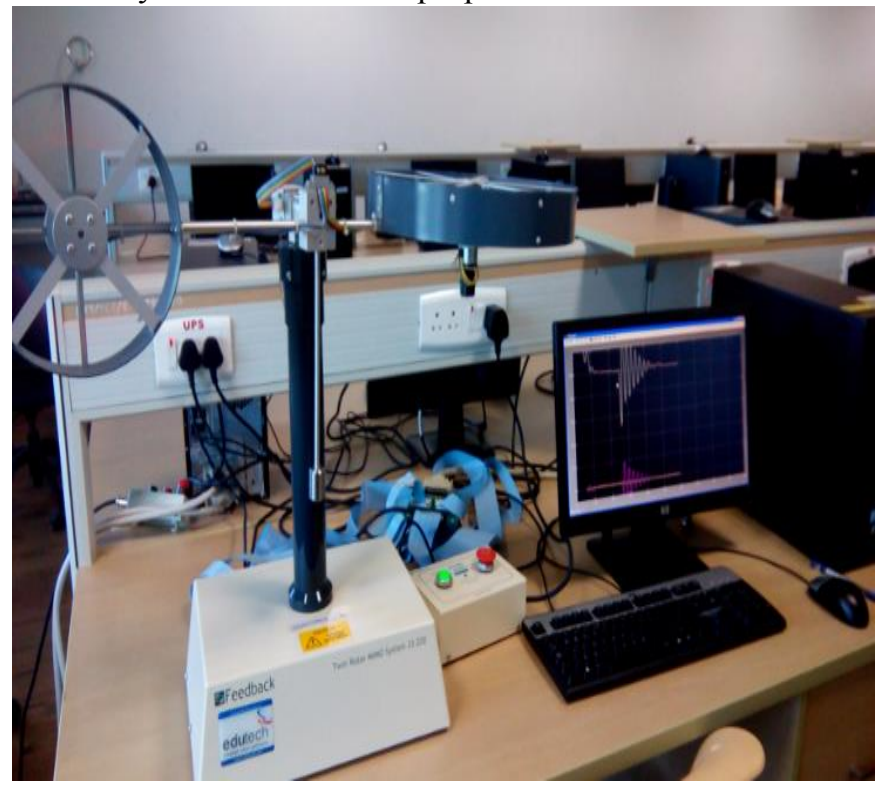

Fig. 2 TRMS Model from Feedback Instruments

Instrument specifications

Line voltage: $230 \mathrm{~V} @ 50 \mathrm{~Hz}$

Consumption: 100 VA.

Weight and Dimensions: $80 \mathrm{~cm}$ x $35 \mathrm{~cm} \times 75 \mathrm{~cm}$

Weight: $11 \mathrm{~kg}$.

The objectives of the proposed project work are as follows:

a. To Estimate the Pitch and Yaw angular positions from the noisy and distorted sensor data obtained from the two optical encoders of the tail and main rotors of the TRMS.

b. Secondary objective is to come up with a comparative study of the various estimation algorithm, in terms of error functions: Integral of Absolute Error (IAE), Integral of Square of Error (ISE) and Integral of Absolute Time error (IATE).

\section{PROBLEM SOLUTION}

The noisy sensor data obtained from the optical sensors of the two rotors are obtained as the input to the estimation block. This initial value of the sensor outputs as well as the control input is used to estimate the next pitch and yaw angles with an appropriate system model. The reference model is used to give the nominal outputs as shown in Figure 3. The first phase of achieving the objective mentioned is by designing estimators for the TRMS model. In the proposed work two different techniques are used for the same. Firstly a model based technique of observer based estimator is designed and later an ANN based estimator is designed.

\section{A. Luenberger Estimator}

The modeling of the system is done using two separate state space representation, one is the pitch model and the other is yaw model. The state space representation of the TRMS system is given by:

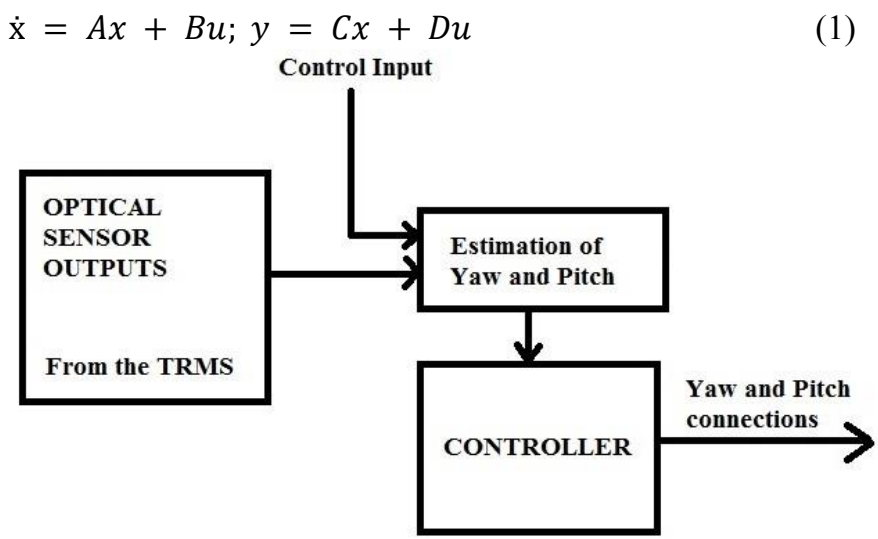

Fig. 3 Block diagram of proposed methodology

The matrices for Pitch Model:

$A=\left(\begin{array}{ccc}-1.4389 & -3.1862 & 1.6706 \\ 0.0803 & -4.9874 & -29.1821 \\ -0.0376 & 0.0474 & -5.5737\end{array}\right) ; B=\left(\begin{array}{l}1 \\ 0 \\ 0\end{array}\right)$

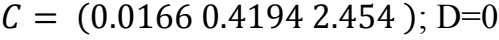

The matrices for Yaw Model:

$A=\left(\begin{array}{ccc}-1.38 & -1.6456 & -14.7611 \\ 0.9244 & -2.5724 & -31.1124 \\ -0.0196 & 0.3346 & -8.0476\end{array}\right) ; B=\left(\begin{array}{l}1 \\ 0 \\ 0\end{array}\right) ;$

$C=\left(\begin{array}{ll}0.001 & 0.0336 \\ 0.4065\end{array}\right) ; \mathrm{D}=0$

The system equation is given as given by equation (1)

The observer equation is given by:

$\hat{x}^{\prime}=A \hat{x}+B u+L *(y-\hat{y})$

$\hat{x}^{\prime}=A \hat{x}+B u+L *(y-C \hat{x})$

$\hat{x}^{\prime}=(A-L * C) \hat{x}+B u+L * y$

$\hat{x}^{\prime}=\tilde{A} \hat{x}+B u+L * y$ 
Where, $\tilde{A}=A-L * C$, and the Eigen values of $\tilde{A}$ control the error dynamics of the observer.

$\mathrm{L}$ is the Luenberger gain and $\hat{x}$ is the estimate of the state $x$ at time $t$.

The poles for the observer for both pitch and yaw are chosen to be at $-1,-2$ and -3

These are the Eigen values of the matrix $\tilde{A}=A-L * C$.

By using Ackermann's Formula

$|(s I-\tilde{A})|=(s+1) *(s+2) *(s+3)$

$|(s I-(A-L * C))|=(s+1) *(s+2) *(s+3)$

Where I is an Identity matrix.

Thus by equating LHS to RHS in equation (8), the Luenberger gain $\mathrm{L}$ is calculated for both the pitch and the yaw and is given below.

The Luenberger gain $L$ for Pitch is: $L=\left(\begin{array}{c}-2.9012 \\ 11.8917 \\ 2.2713\end{array}\right)$

The Luenberger gain L for Yaw is: $L=\left(\begin{array}{l}35.3716 \\ 76.5373 \\ 19.7974\end{array}\right)$

\section{A. Neural Network Estimator}

To design the estimator for finding the yaw and pitch neural network algorithms are made use. Neural network algorithm is process of finding the unknown yaw and pitch by using a predetermined output. The set these predetermined data is called training data and the method is called training. Training data consist of input data and target data, in the proposed work signal to the main rotor and tail rotor is called the input data and the yaw and pitch corresponding to the signal is called target data. For training $150 \times 2$ samples were used, these were further divided into training data, validation and testing data with the ratio of $70 \%, 15 \%$ and $15 \%$ corresponding. Estimation based on ANN is based on a time series correlation between the input and the output, also called as the targets. This assumes a black body model, where the dynamics of the system are not known and the forecasting is done only from the input output relationship of the system. In the proposed work back propagation based neural network algorithm consisting of two hidden layers with $6 \times 2$ and $4 \times 2$ neurons respectively as shown in Figure 4 [27], [28]. Network parameter of the proposed neural network is given in Table 1 .

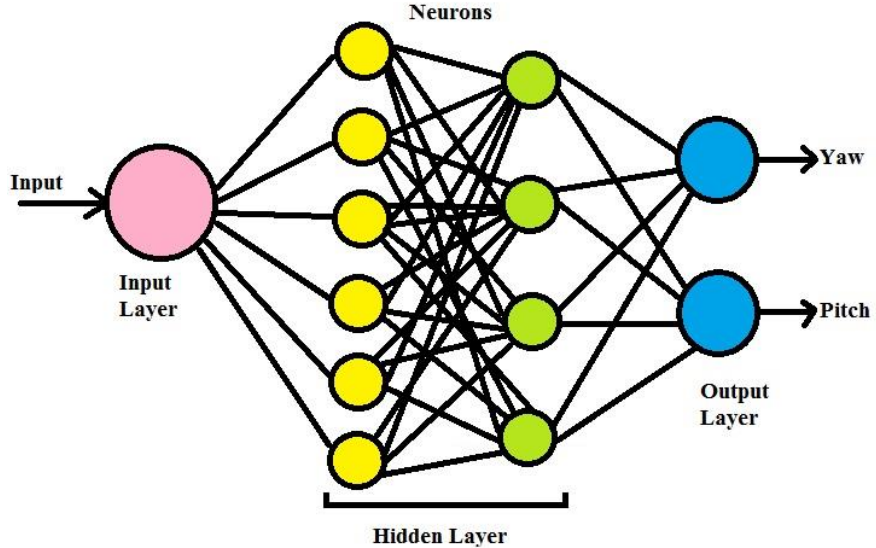

Fig. 4 Structure of neural network model

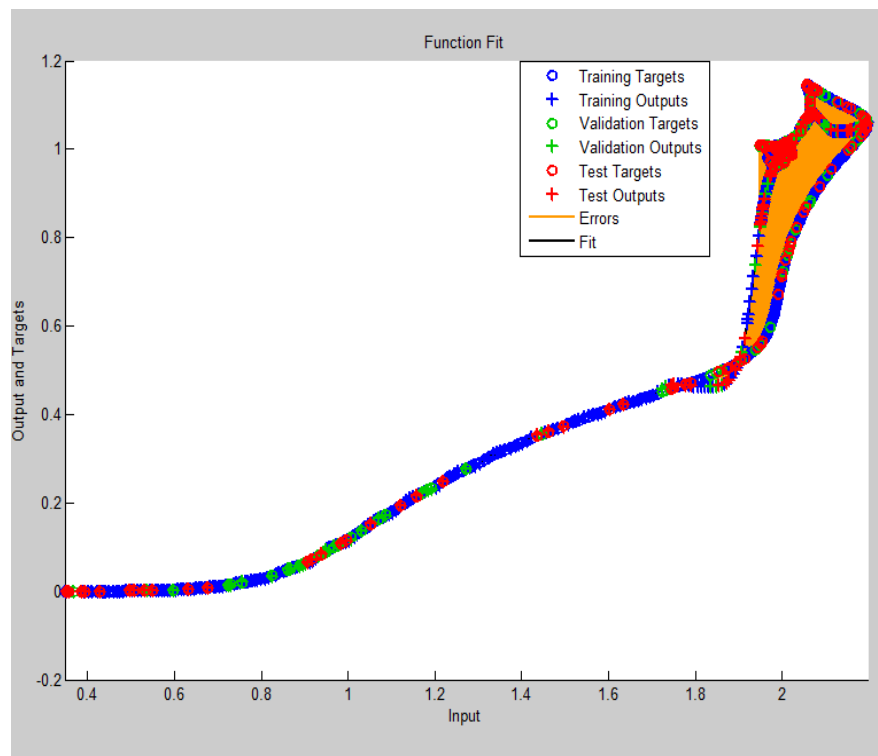

Fig. 5 Plot of fit graph for pitch

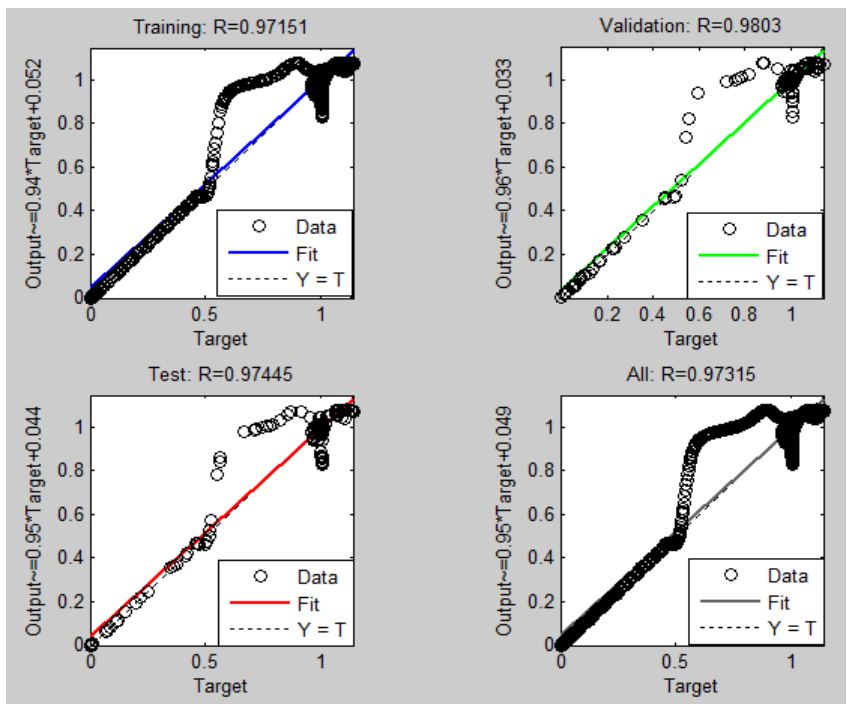

Fig. 6 Regression graph for pitch training 


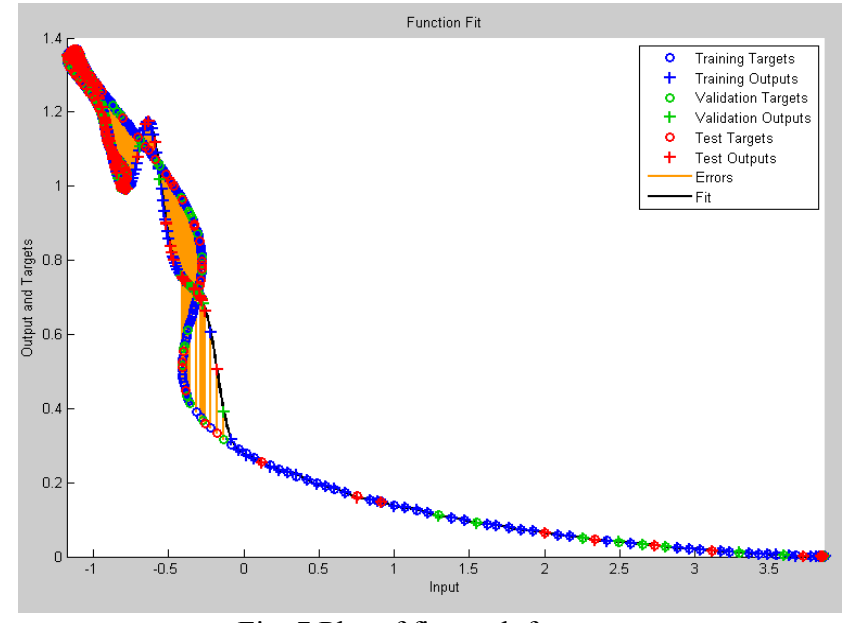

Fig. 7 Plot of fit graph for yaw
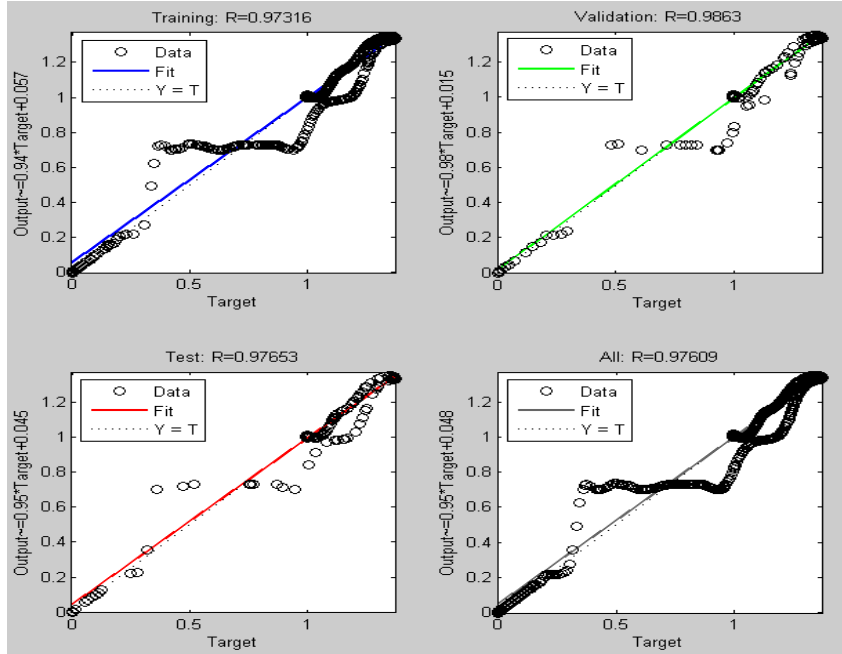

Fig. 8 Regression graph for pitch training.

TABLE I. Neural network parameters

\begin{tabular}{|l|l|c|}
\hline \multicolumn{2}{|c|}{ OPTIMIZED PARAMETERS OF THE NEURAL NETWORKS } \\
MODEL
\end{tabular}

Here the neurons are trained to replicate the behavior of the system by using the given set of data. The back propagation model with a size of 20 neurons are used to predict the output. The plot fit graph and regression graph obtained after training for pitch and yaw is shown in Figure 5, Figure 6, Figure 7 and Figure 8 respectively.

\section{RESULTS AND ANALYSIS}

Once the estimators are designed using Luenberger and $\mathrm{ANN}$, it is subjected to test individually to analysis the performance. For analyzing the performance it is subjected to test with unit input condition and then with disturbance. Further the error constant analysis is carried on for evaluation of performance based on standard error constants.

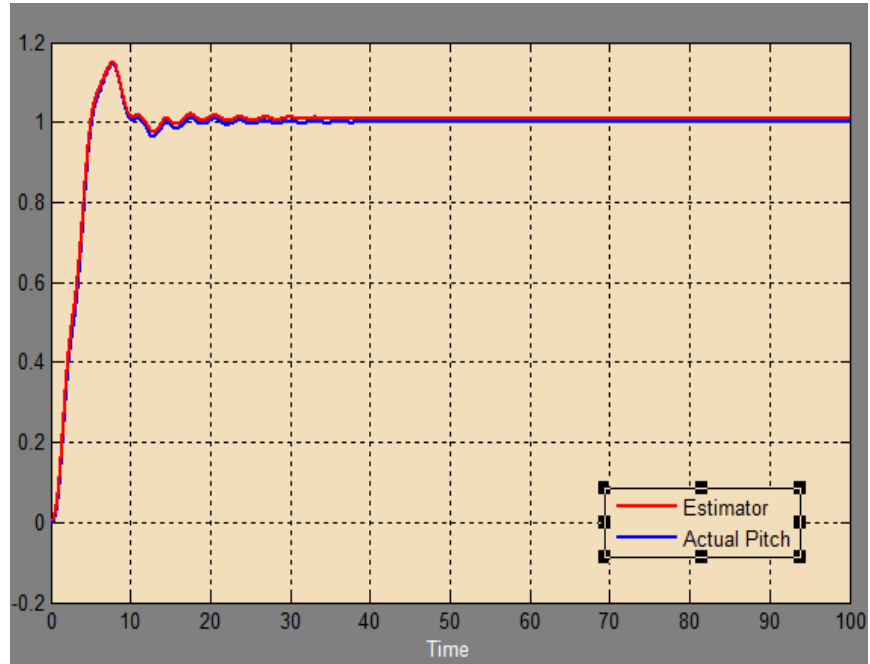

Fig. 9 Plot of tracking pitch by designed observer for unit input.

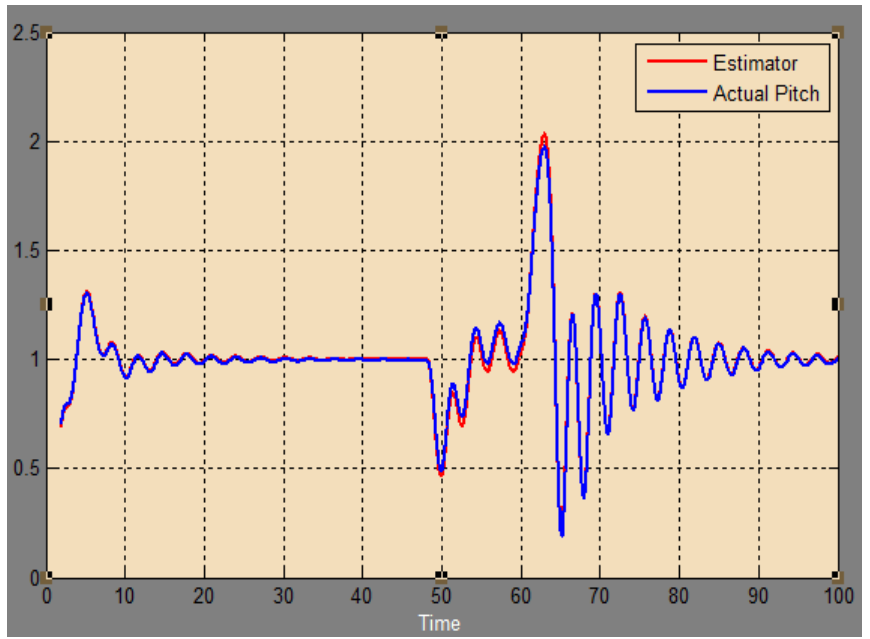

Fig. 10 Plot of tracking pitch by designed observer for disturbance. 


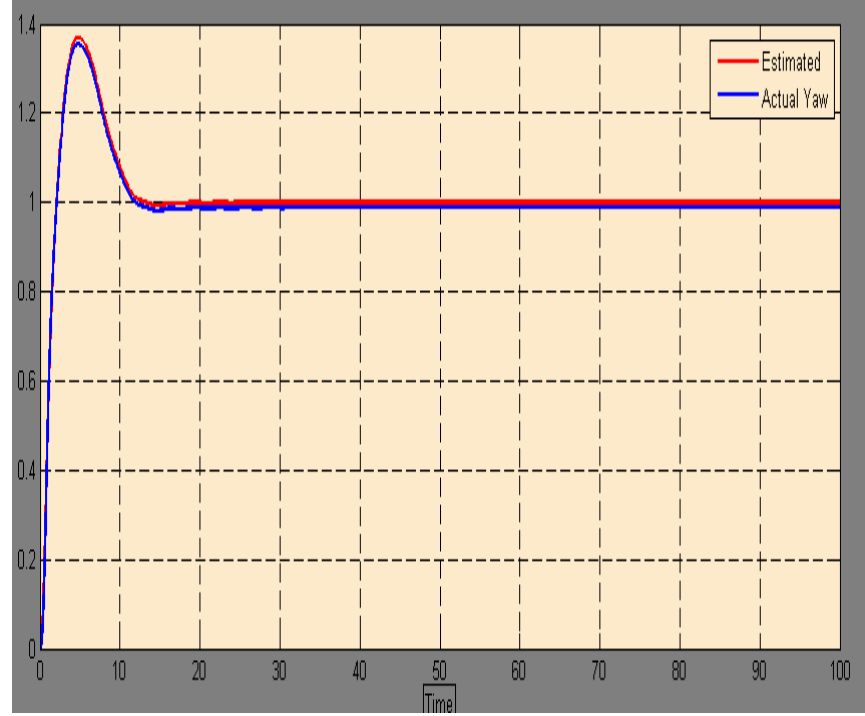

Fig. 11 Plot of tracking yaw by designed observer for unit input.

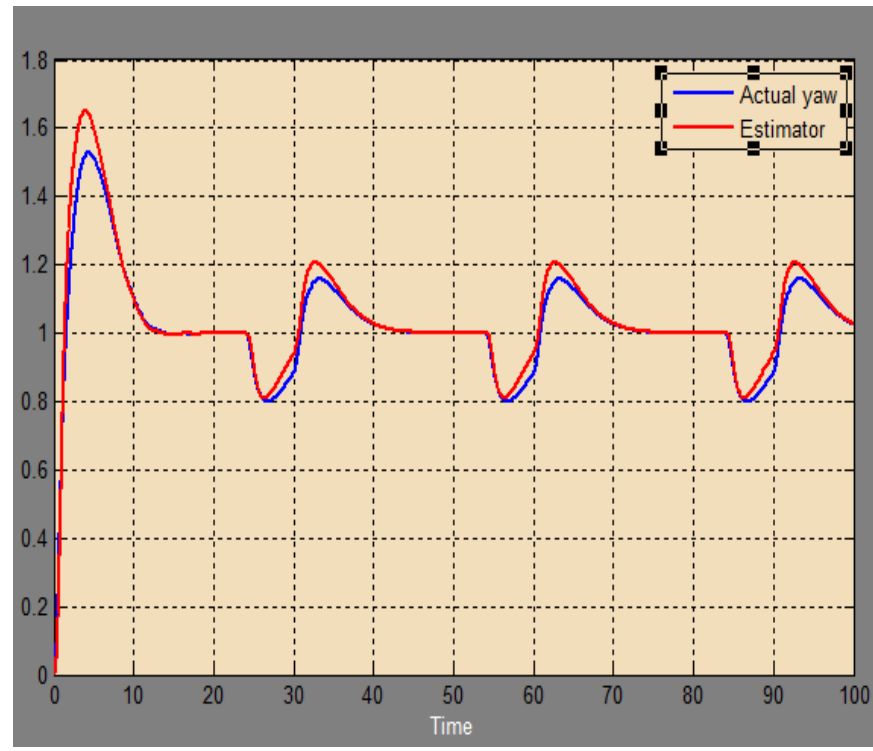

Fig. 12 Plot of tracking yaw by designed observer for unit input.

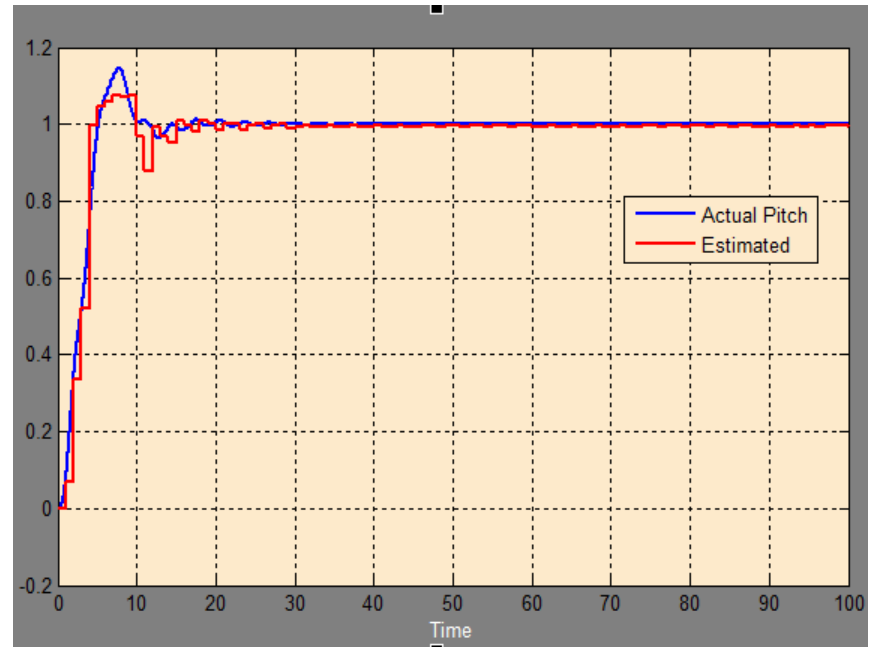

Fig. 13 Plot of tracking yaw by designed observer for unit input.

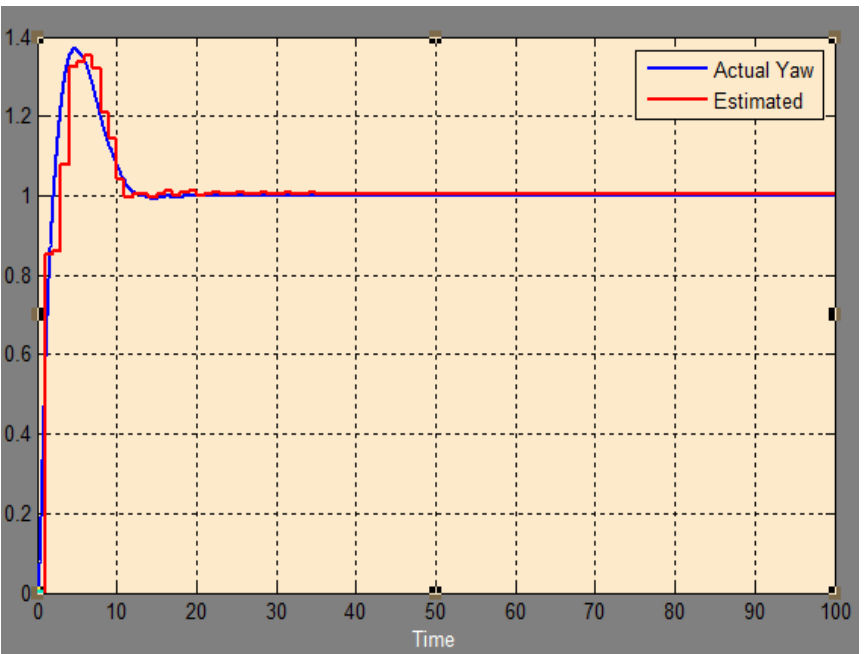

Fig. 14 Plot of tracking yaw by designed observer for unit input.

Response of designed observer based system is plot in Figure 9, Figure 10, Figure 11, Figure 12, Figure 13and Figure 14 for step input. Response shows that the system was able to track the actual pitch and yaw accurately. Tracking response with disturbance is also plot. From Figure 9 to and Figure 14 it is clear that the observer based estimator was able to track accurately. Similarly, tracking output for ANN based estimator is shown in Figure 15 and Figure 16, the output shows the ANN based estimator was also able to track the pitch and yaw accurately. To analyze the complete behavior in terms of error the IAE, ISE, and ITAE values are computed.

$$
\begin{aligned}
& I S E=\int e^{2} d t \\
& I A E=\int|e| d t \\
& I T A E=\int t|e| d t \\
& \text { Where e - error } \\
& \mathrm{t}-\text { Time taken }
\end{aligned}
$$

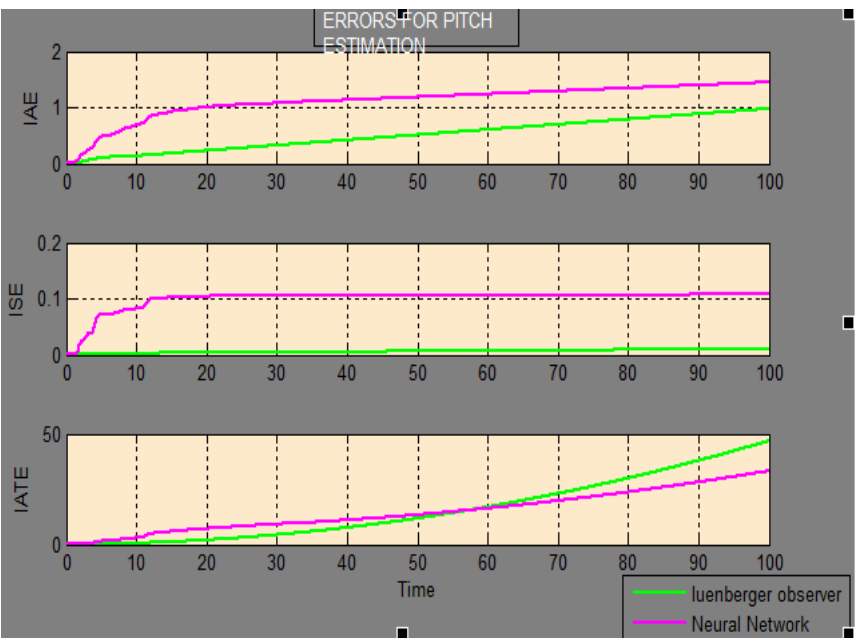

Fig. 15 Comparison for ISE, IAE, and ISTE for Pitch estimation 


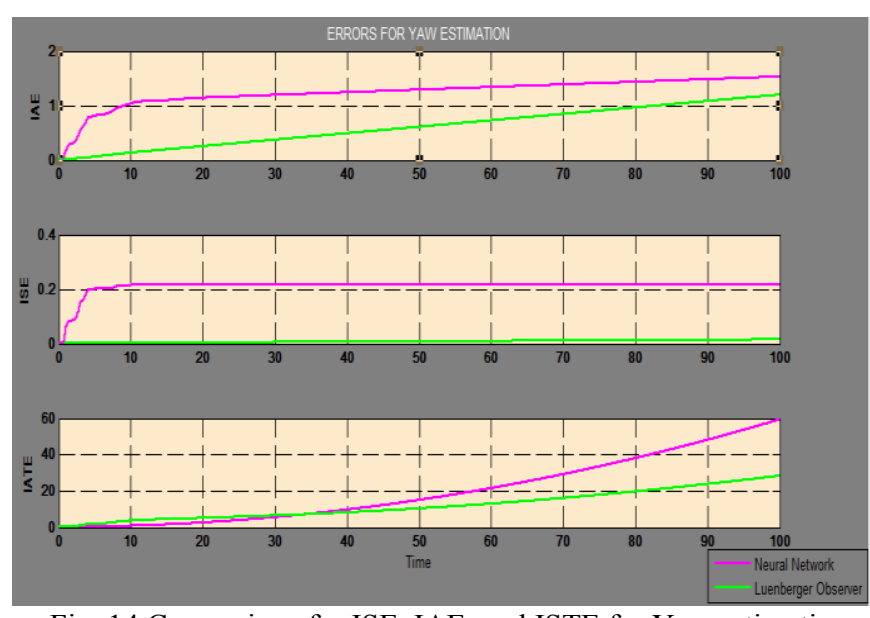

Fig. 14 Comparison for ISE, IAE, and ISTE for Yaw estimation

Overall performance can be checked from IAE since this penalizes all kinds of error. ISE penalizes large errors more heavily hence this can be used to check transient errors or errors during oscillatory phase. ITAE penalizes errors that occur after certain time more heavily, hence the errors in steady state are more amplified than the errors in transient phase. The comparative results of all these errors for pitch and yaw are shown in Figure 15 and Figure 16 respectively and Table 2.

TABLE II. Computation of error for yaw and pitch estimation at time $=50$ seconds

\begin{tabular}{|l|l|l|l|l|}
\hline \multirow{2}{*}{} & \multicolumn{2}{|c|}{ Pitch estimation } & \multicolumn{2}{c|}{ Yaw estimation } \\
\cline { 2 - 5 } & Observer & ANN & Observer & ANN \\
\hline IAE & 0.5086 & 1.18 & 0.69 & 1.28 \\
\hline ISE & 0.005 & 0.11 & 0.04 & 0.22 \\
\hline IATE & 11.745 & 13.35 & 9 & 10.3 \\
\hline
\end{tabular}

\section{CONCLUSION}

Twin rotor multi input multi output system consists of a coupled system, mimicking the behavior of helicopter. The model helps us to understand the control behavior so as to maintain parameters like pitch yaw and roll. To analyze the behavior it is primarily essential to monitor the system, for monitoring we need to install the sensory system. The paper reported a technique for design and comparison of designed estimator for computation of pitch and yaw in case of TRMS. In the reported work two technique were one with computation of estimator based on observer and other based on ANN. From the graphs shown in Figure 9 to Figure 16 it is seen that both the estimators were able to track pitch and yaw accurately. But it was seen that the observer based technique was giving high error during transients and very low errors when it settled. But in case of ANN though the error during transients was low as compared to that of observer its error during static conditions was high.

The analysis helps to understand the dynamic behavior of the TRMS system for yaw and pitch variation, which can be further extended for roll monitoring as well. The work presented would help to diagnose the fault in monitoring the variables using physical system.

\section{References}

[1] A. Rahideh, H. M. Shaheed and A. H. Bajodah, "Adaptive Nonlinear Model Inversion Control of a Twin Rotor MIMO System using Artificial Intelligence," Proceedings of the Institution of Mechanical Engineers, Part G: Journal of Aerospace Engineering, vol. 221, no. 3, pp. 343-351, Mar. 2007.

[2] J. Jih-Gau, H. Ming-Te, and L. Wen-Kai, "PID control using presearched genetic algorithms for a MIMO system," IEEE Transactions on Systems, Man, and Cybernetics, Part C (Applications and Reviews), vol. 38, no. 5, pp. 716-727, Sep. 2008.

[3] D. Xiucheng, Z. Xiaoxiao, and Mei Shu, "Research of control method based on 3-DOF twin rotor MIMO system," In 2008 7th World Congress on Intelligent Control and Automation, pp. 3279-3283, Jun. 2008.

[4] E. Besada-Portas, J.A. Lopez-Orozco, J. Aranda and J.M. de la Cruz, "Virtual and remote practices for learning control topics with a 3DOF quadrotor," 10th IFAC Symposium Advances in Control Education, The International Federation of Automatic Control, vol. 46, no. 17, pp. 28-30, Aug. 2013.

[5] R. Rashad, A. El-Badawy and A. Aboudonia, "Sliding mode disturbance observer-based control of a twin rotor MIMO system," ISA Transactions, vol. 69, pp. 166-174, Jul. 2017.

[6] P. Marcin, M. Witczak, C. Aubrun, and J. Korbicz, "Simultaneous estimation of multiple sensor and process faults for non-linear discrete-time systems," IFACPapersOnLine, vol. 51, no. 24, pp. 82-87, Jan. 2018.

[7] R. Damiano, F. Nejjari, and V. Puig, "Shifting linear quadratic control of constrained continuous-time descriptor LPV systems," IFAC-PapersOnLine, vol. 48, no. 26, pp. 25-30, Jan. 2015.

[8] R. Raul-Cristian, P. Radu-Emil, and D. Radu-Codrut, "Second order intelligent proportional-integral fuzzy control of twin rotor aerodynamic systems," Procedia computer science, vol. 139, pp. 372-380, Jan. 2018.

[9] V. Joost, C. W. Scherer, and H. Köroğlu, "Robust stability and performance analysis based on integral quadratic constraints," European Journal of Control, vol. 31, pp. 1-32, Sep. 2016.

[10] A. Roohallah, M. Tavakoli-Kakhki, A. K. Sedigh, and A. Fatehi, "Robust fractional order PI controller tuning based on Bode's ideal transfer function," IFAC-PapersOnLine, vol. 49, no. 9, pp. 158-163, Jan. 2016.

[11]M. Alexey, I. Furtat, and A. Kremlev, "Robust control of twin rotor MIMO system with quantized output," IFACPapersOnLine, vol. 50, no. 1, pp. 4849-4854, Jul. 2017.

[12]S. Zeghlache, and $\mathrm{N}$ Amardjia, "Real time implementation of nonlinear observer-based fuzzy sliding mode controller for a twin rotor multi-input multi-output system (TRMS)," Optik, vol. 156, pp. 391-407, Mar. 2018. 
[13]R. Damiano, F. Nejjari, and V. Puig, "Quasi-LPV modeling, identification and control of a twin rotor MIMO system," Control Engineering Practice, vol. 21, no. 6, pp. 829-846, Jun. 2013.

[14] Y. Bouzid, H. Siguerdidjane, and Y. Bestaoui, "Nonlinear internal model control applied to VTOL multi-rotors UAV," Mechatronics, vol. 47, pp. 49-66, Nov. 2017.

[15]L. Shi, and L. Yue-Yang, "Neural network based nonlinear model predictive control for an intensified continuous reactor," Chemical Engineering and Processing: Process Intensification, vol. 96, pp. 14-27, Oct. 2015.

[16] S. S. Butt and H. Aschemann, "Multi-variable integral sliding mode control of a two degrees of freedom helicopter," IFAC-PapersOnLine, vol. 48, no. 1, pp. 802807, Jan. 2015.

[17] R. Raul-Cristian, R. Mircea-Bogdan and P. Radu-Emil. "Multi-input-multi-output system experimental validation of model-free control and virtual reference feedback tuning techniques." IET Control Theory \& Applications 10, no. 12, pp. 1395-1403, Aug. 2016.

[18] J. C. Salazar, P. Weber, R. Sarrate, D. Theilliol and F. Nejjari, "MPC design based on a DBN reliability model: Application to drinking water networks," IFACPapersOnLine, vol. 48, no. 21, pp. 688-693, Jan. 2015.

[19] S. N. Pawar, R. H. Chile, and B. M. Patre, "Modified reduced order observer based linear active disturbance rejection control for TITO systems," ISA transactions, vol. 71, pp. 480-494, Nov. 2017.

[20] L. Shi, and L. Yueyang, "Model predictive control of an intensified continuous reactor using a neural network Wiener model," Neurocomputing, vol. 185, pp. 93-104, Apr. 2016.

[21] P. Chalupa, J. Přikryl, and J. Novák, "Modelling of twin rotor MIMO system," Procedia Engineering, vol. 100, pp. 249-258, Jan. 2015.

[22]N. K. Arun and B. M. Mohan, "Modeling and Computational Aspects of Nonlinear Fuzzy TITO PI/PD Controller via Height Defuzzification," IFACPapersOnLine, vol. 51, no. 1, pp. 347-352, Dec. 2018.

[23] P. Radu-Emil, R. Mircea-Bogdan, R. Raul-Cristian, and E. M. Petriu, "Model-free sliding mode control of nonlinear systems: Algorithms and experiments," Information Sciences, vol. 381, pp. 176-192, Mar. 2017.

[24]A. Dòria-Cerezo, L. Van Der Heijden, and J. MA Scherpen, "Memristive port-Hamiltonian control: Pathdependent damping injection in control of mechanical systems," European Journal of Control, vol. 19, no. 6, pp. 454-460, Dec. 2013.

[25] R. Damiano, A. Cristofaro, K. Gryte and J. Tor Arne, "LPV model reference control for fixed-wing UAVs,"
IFAC-PapersOnLine, vol. 50, no. 1, pp. 11559-11564, Jul. 2017.

[26] Pandey Samit Kumar, Control of Twin Rotor Mimo System, LAP Lambert Academic Publishing, 2015. ISBN: 978-3659760402

[27] Faghri, Ardeshir, and Aneja Sandeep. "Analysis of Performance of Backpropagation ANN with Different Training Parameters." Neural networks in transport applications. Routledge, pp. 57-84, 2019.

[28] Aggarwal, Charu C, Neural Networks and Deep Learning, Springer International Publishing, 2019, ISBN: 978-3319-94463-0

Santhosh K V received his Bachelors and Masters from Visvesvaraya Technological University, Belgaum in 2004 and 2006. He received his $\mathrm{PhD}$ from National Institute of Technology Silchar in the year 2013. Dr. Santhosh KV is currently working as Associate Professor, in the Department of Instrumentation and Control Engineering, Manipal Institute of Technology, MAHE. Dr. Santhosh KV research interest are in the area of sensor fusion, smart sensor, and fault detection and isolation.

Preeti Mohanty was born in Manipal, Karnataka on 29th January, 1986. She received her graduation in Electronics and Telecommunication from Gandhi Institute of Engineering and Technology, Odisha, India in the year 2007, and postgraduation in Digital Electronics and Advanced Communication from Manipal Institute of Technology, Manipal, Karnataka, India in the year 2009. She is presently working in Manipal Institute of Technology as Assistant Professor (Senior Scale) in the Department of Instrumentation and Control Engineering. Her research area are in the field of Digital electronics, Sensor design and Neural network

\section{Creative Commons Attribution License 4.0 (Attribution 4.0 International, CC BY 4.0)}

This article is published under the terms of the Creative Commons Attribution License 4.0 https://creativecommons.org/licenses/by/4.0/deed.en_US 